\title{
V020 A 3D OCEAN BOTTOM CABLE REPEATABILITY STUDY
}

CRAIG J. BEASLEY, RON E. CHAMBERS, RICK L. WORKMAN, KEN CRAFT and LAURENT J. MEISTER Western Atlas International Inc., PO Box 2469, Houston TX 77252-2469, USA

\section{Introduction}

There has been a resurgence of interest lately in using repeated seismic surveys as a reservoir description and production monitoring tool. Although studies of repeated seismic surveys for mapping steam fronts go back more than 10 years, recent advances have extended time lapse or 4-D seismic to map gas-oil and oil-water contacts with significant economic benefit (Greaves and Fulp, 1987; Pullin et al., 1987; Dunlop et al., 1991; Johnstad et al., 1995). Recent studies, e.g., Watts, 1995, have made fortunate use of legacy 3-D data not originally acquired for a 4-D study and found that the varying quality of the different vintages of 3-D data was a factor limiting the resolution of their studies. As a result, the question arises how best to carry out the data acquisition and processing for a 4-D seismic program so that the data are repeatable to the extent that differences are attributable to movement of the reservoir fluids rather than acquisition and processing.

In this paper we present a case study of the repeatability of the ocean bottom cable (OBC) system based on two colocated 3-D surveys acquired and processed independently. First we compare off-the-shelf 3-D migrated data volumes. Then, beginning with migration, we back up in the processing sequence until we arrive at analogous flows for the two surveys. We found that in this case it was essential to reprocess the data from the prestack stage to account for known differences to achieve the best results.

\section{Acquisition}

Two OBC surveys were carried out with a separation of four months over an area that contained no known hydrocarbon production or other subsurface mechanism for changing the seismic response. The survey plan was identical for the two surveys, but for a variety of reasons, such as varying currents and wind, the receiver positions were not identical between the two surveys. Nevertheless, the flexibility of the $\mathrm{OBC}$ method allowed changes in receiver positions to be offset by corresponding changes in shot locations yielding final fold, offset, and azimuth distributions that were very similar. The same recording instruments were used for the two surveys with one significant difference: while one survey employed a $3 \mathrm{~Hz}$ instrument low cut filter, the other used an $8 \mathrm{~Hz}$ filter. As we shall see, dealing with such seemingly minor differences in the initial stages of processing is key to insuring repeatability.

\section{Off-the-shelf 4-D}

In some areas with extensive 3-D coverage, existing 3-D surveys overlap and thus the appealing possibility arises to do 4-D seismic simply by studying the existing different surveys. To test the notion of such raw repeatability, we began our investigation with the existing data which were processed with similar, but independent sequences. Adding to the acquisition differences noted above, velocities were interpreted independently, reflection statics, though small, had been derived from and applied to one data volume but not the other, and, although identical migration algorithms were used, the migration velocity fields and aerial extent of the migrated volumes were different. It is fair to ask, as have some of our colleagues, if one should even attempt to difference these data, but, it is salient in light of reported use of legacy data in 4-D without reprocessing specifically for the 4-D project. We subtracted the two off-the-shelf data volumes after applying random noise attenuation (RNA) and a single timevarying scale function and found the differences to be on the order of magnitude of the data itself.

Next we applied a matching filter as is common in the literature on 4-D seismic to account for systematic differences in the data. A single matching filter derived over a $1500 \mathrm{~ms}$ window reduced the differences near the 
filter design location but increased differences elsewhere. Further poststack investigations revealed that remigration with identical velocity fields and aerial extent, improved agreement somewhat but still yielded substantial differences. Clearly, prestack reprocessing was indicated.

\section{Prestack reprocessing}

Two main factors were first considered in the prestack reprocessing: velocity and statics. Surprisingly, we found that stacking with the same velocities and static treatment yielded only incrementally improved results which led us to review the entire processing sequence. We first applied a shaping filter to equalize the instrument responses and bandpass filtered the data. Next we computed offset-consistent amplitude factors: for each common-offset gather, single time-varying scale functions were derived to balance the traces prior to further processing. Afterwards, identical processing sequences were applied to the data through stack. As above, a single matching filter was derived after application of RNA and balancing, and yielded differences that were small compared to the original data.

\section{Discussion and conclusions}

At first glance, the use of powerful processing steps such as RNA and matching filters may cause concern. However, RNA removes only noise that is spatially random and, as used here, is common practice. Matching filters can be a concern if allowed to vary significantly or if they are influenced by the reservoir zone. In any case, such measures are likely to be necessary in 4-D processing. In our studies, the effects of noise play a major role in observed differences. In particular, adaptive processes are influenced by noise and should be avoided until the noise has been addressed.

We have shown in this particular example that the OBC system produced data with a high degree of repeatability provided the data are processed from the prestack stage with repeatability as a goal. We also found it essential to account for known differences such as instrument responses and noise characteristics at the prestack stage. Perhaps the best argument that conventional seismic systems are useful for 4-D is the growing body of literature that indicates successful use of legacy data acquired with varying acquisition systems and designs.

\section{References}

Dunlop, K.N.B., King, G.A., and Breitenbach, E.A., 1991, Monitoring of oil/water fronts by direct measurement: Journal of Petroleum Technology, 43, 596-602.

Greaves, R.J., and Fulp, T.J., 1987, Three-dimensional seismic monitoring of an enhanced oil recovery process: Geophysics, 52, 1175-1187.

Johnstad, S.E., Seymour, R.H., and Smith, P.J., 1995, Seismic reservoir monitoring over the Oseberg field during the period 1989-1992: First Break, 13, 169-183.

Pullin, N.E., Jackson, R.K., Matthews, L.W., Thorburn, R.F., Hirsche, W.K., and den Boer, L.D., 1987, 3-D seismic imaging of heat zones at an Athabasca tar sands thermal pilot: 57th Ann. Internat. Mtg., Soc. of Expl. Geophys., Expanded Abstracts, 391-394.

Watts, G., Jizba, D., Gawith, D., and Gutteridge, P., 1995, Reservoir monitoring on the Magnus field through 4-D time-lapse seismic analysis: 57th Ann. Mtg., Eur. Assoc. Geoscien. Eng., Expanded Abstracts, F013. 